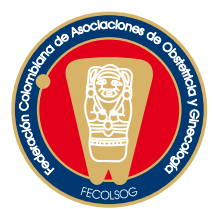

INVESTIGACIÓN ORIGINAL

\title{
ESCALA DE EDINBURGH PARA DEPRESIÓN POSPARTO: CONSISTENCIA INTERNA Y ESTRUCTURA FACTORIAL EN MUJERES EMBARAZADAS DE CARTAGENA, COLOMBIA
}

\section{Validating the Edinburgh Postnatall Depression Scale's internal consistency and factor structure} amongst pregnant women in. Cartagena, Colombia Adalberto Campo-Arias, M.D., * Cristian Ayola-Castillo**, Henry Miguel Peinado-Valencia**, Maribel Amor-Parra**, Zuleima Cogollo***

Recibido: julio 5/07 - Revisado: noviembre 13/07 - Aceptado: noviembre 14/07

\section{RESUMEN}

Objetivos: determinar la consistencia interna y la estructura de factores de la escala de Edinburgh para depresión posparto en mujeres embarazadas de Cartagena, Colombia.

Materiales y método: se diseñó un estudio de validación de un instrumento de tamizaje sin patrón de oro (metodológico), de la escala de Edinburgh para depresión posparto con 200 mujeres gestantes letradas entre 18 y 42 años, con un promedio de 24,3 años $(\mathrm{DE}=5,2)$, residentes de estrato económico bajo que asistían a consulta externa en una clínica pública de maternidad en Cartagena, Colombia. Se determinó consistencia interna (alfa de Cronbach) y la estructura factorial de la escala (análisis factorial exploratorio).

Resultados: la escala mostró una consistencia interna de 0,78, una estructura tridimensional (ánimo

\footnotetext{
Médico psiquiatra, Director de Investigaciones, Grupo de Investigación del Comportamiento Humano, Instituto de Investigación del Comportamiento Humano, Bogotá, y profesor asociado, Facultad de Enfermería, Universidad de Cartagena. Correspondencia: Universidad de Cartagena, Área de la Salud, Facultad de Enfermería, Campus de Zaragocilla, Cartagena, Colombia. Teléfono y fax 566981 81. Correo electrónico: acampoa@unicartagena. edu.co o campoarias@comportamientohumano.org

** Estudiantes, Facultad de Enfermería, Universidad de Cartagena.

*** Enfermera, profesora titular, Facultad de Enfermería, Universidad de Cartagena.
}

deprimido, anhedonia y desesperanza y culpa) que explicaba el 59,9\% de la varianza.

Conclusiones: el estudio muestra que la escala de Edinburgh para depresión posparto posee una alta consistencia interna y una estructura tridimensional para el concepto de depresión en mujeres embarazadas de Cartagena, Colombia.

Palabras clave: consistencia interna, estructura factorial, depresión, embarazo, escala de Edinburgh para depresión posnatal, estudios de validación.

\section{SUMMARY}

Objectives: determining the Edinburgh Postnatal Depression Scale's internal consistency and factor structure amongst pregnant women in Cartagena, Colombia.

Materials and method: this was a validation study (lacking a gold-standard) designed for use with 200 literate, pregnant women aged 18 to 42 (24.3 average age, 5.2 SD) living in lowincome neighborhoods and attending a public obstetric clinic in Cartagena, Colombia. Internal consistency (Cronbach's alpha) and factor structure (exploratory factor analysis) of the Edinburgh 
Postnatal Depression Scale were computed.

Results: the Edinburgh Postnatal Depression Scale presented 0.78 internal consistency and a threedimensional structure (depression, anhedonia and a hopeless-guilty mood) explaining 59.9\% of variance.

Conclusions: the study showed that the Edinburgh Postnatal Depression Scale had high internal consistency and was composed of a threedimensional structure exploring the concept of depression in low-income pregnant women in Cartagena, Colombia.

Key words: internal consistency, factorial structure, depression, pregnancy, Edinburgh Postnatal Depression Scale, validation study.

\section{INTRODUCCIÓN}

Los trastornos depresivos representan un problema importante de salud pública. ${ }^{1}$ Estos se observan con mayor frecuencia en la población femenina que en la masculina. ${ }^{2}$ Además, la frecuencia de estos trastornos parece ser sustancialmente mayor durante el embarazo y el puerperio que durante otras épocas del ciclo vital, ${ }^{3-5}$ especialmente en embarazadas de bajos ingresos económicos. ${ }^{6}$

La presencia de trastorno depresivo mayor, con frecuencia abreviado o llamado simplemente como depresión, durante el embarazo, incrementa en forma importante la morbilidad y mortalidad tanto para la madre como para el fruto del embarazo, es decir, se asocia a conductas poco saludables como el omitir la atención prenatal o el uso de sustancias nocivas (tabaco, alcohol, sustancias ilegales). De la misma forma, el trastorno depresivo mayor en gestantes es un factor de riesgo para intentos de suicidios y suicidio consumados. ${ }^{7}$ Así mismo, la presencia de este trastorno en el embarazo aumenta el riesgo de presentar preeclampsia, partos prematuros y productos de bajo peso al nacer. ${ }^{8}$ Además, representa para la gestante un riesgo elevado de presentar un episodio depresivo durante el posparto. ${ }^{9,10}$ Finalmente, se observa que las madres con un menor apego a los hijos son aquellas que informaron más síntomas depresivos y de ansiedad durante el embarazo. ${ }^{11}$

Varias escalas se usan para identificar trastornos depresivos durante la gestación. Sin embargo, la escala para depresión del centro epidemiológico (CES-D, por sus siglas en inglés) ${ }^{12}$ y la escala de Edinburgh para depresión posparto (EPDS, por sus siglas en inglés) son las más conocidas y usadas con este propósito. ${ }^{13}$ Es importante tener presente que la escala de Edinburgh para depresión posparto se diseñó para cuantificar síntomas depresivos cognoscitivos exclusivamente en el posparto. Se excluyeron sistemáticamente algunos síntomas físicos relacionados con un episodio depresivo que pueden estar presentes durante el embarazo y considerarse como propios de la gestación. ${ }^{14}$ No obstante, se utiliza igualmente en la detección de estos síntomas durante la gestación. ${ }^{15,16}$

Es importante conocer primero el comportamiento psicométrico de una escala con una única aplicación y sin criterio de referencia (la consistencia interna y la estructura factorial) en diferentes poblaciones, dado que el comportamiento de las escalas varía de acuerdo con la población estudiada. Una aceptable confiabilidad y validez de una escala en una población determinada no garantizan un adecuado comportamiento en otro grupo con alguna otra característica distintiva y pueden invitar a que se hagan algunas modificaciones o adaptaciones para conseguir un comportamiento comparable y aceptable. ${ }^{17}$

Para determinar la confiabilidad inicial de una escala se explora la consistencia interna, ${ }^{18,19}$ lo que se refiere a que los ítems que conforman la escala tienen adecuados niveles de correlación entre ellos. Si existe una adecuada consistencia interna, se considera que la escala es homogénea y sugiere indirectamente que los ítems tienen mucho en común. ${ }^{17}$ Para conocer los factores o dimensiones latentes en una escala homogénea se utiliza el análisis de factores. Los factores o dimensiones son, a su vez, pequeñas agrupaciones de los ítems de la escala. Igualmente, el análisis de factores es una de las estrategias que 
se usa para conocer la validez del constructo de una escala. ${ }^{20}$ La validez del constructo evalúa hasta qué punto el indicador o la definición operativa mide el concepto. ${ }^{21}$

En relación con la consistencia interna de la escala de Edinburgh para depresión posparto en mujeres en esta etapa, Cox y cols. informaron un coeficiente $0,87 .{ }^{13}$ Mientras que en estudios realizados con embarazadas, la escala de Edinburgh para depresión posparto mostró una consistencia interna entre 0,76 y $0,85^{22-25}$ y una estructura factorial de dos factores que explicaban más del $50 \%$ de la varianza. ${ }^{23-25}$

El objetivo general de este estudio fue determinar la consistencia interna (homogeneidad) y la estructura de factores (dimensionalidad) de la escala de Edinburgh para depresión posparto en mujeres embarazadas residentes en estrato bajo en Cartagena, Colombia.

\section{MATERIALES Y MÉTODOS}

El presente es un estudio metodológico para validar una escala o instrumento sin patrón de oro, con una sola aplicación del instrumento, con un diseño transversal, ${ }^{26}$ en embarazadas del área urbana de Cartagena, ciudad ubicada en la costa norte de Colombia, que asistían al control prenatal en una clínica pública de maternidad durante el mes de abril del 2007.

El Comité de Ética de la Universidad de Cartagena revisó y aprobó la propuesta de investigación. Se solicitó la autorización del representante legal de la clínica y la participación de las pacientes fue completamente voluntaria, después que se les informó las características del estudio, del mínimo riesgo que implicaba y del mantenimiento del anonimato, de acuerdo con la Declaración de Helsinki ${ }^{27}$ y las disposiciones colombianas para investigaciones en salud. ${ }^{28}$

Se tomó una muestra no probabilística de 200 mujeres embarazadas. Para alcanzar el objetivo de la investigación se precisaba de una muestra que podía oscilar entre 100 y 200 participantes, es decir, entre 10 y 20 participantes por ítem. ${ }^{17}$ Participaron embarazadas mayores de 18 años, con o sin problemas patológicos, residentes de estrato bajo del área urbana de Cartagena, pertenecientes al régimen subsidiado, que asistían a control prenatal en una institución de segundo nivel de atención. Se incluyeron por conveniencia solo pacientes de estrato bajo porque representan más del 90\% de las personas atendidas en la institución y se esperaba contar con una muestra lo suficientemente homogénea. Se excluyeron las embarazadas iletradas o aquellas que presentaban signos evidentes de déficit cognoscitivo que les impedía responder por sí mismas el cuestionario.

Esta muestra se completó después de solicitar la participación de 260 embarazadas (76,9\%); 47 embarazadas no reunieron los criterios de inclusión (20 no vivían en Cartagena, 20 eran menores de edad y 7 eran iletradas) y 13 se negaron a participar. La edad de las 200 participantes se encontraba entre 18 y 42 años con un promedio de 24,3 años $(\mathrm{DE}=5,3)$; el $73 \%$ eran católicas y $27 \%$ evangélicas; el 90,5\% de las participantes tenía pareja estable; la escolaridad osciló entre 2 y 15 años, con un promedio de 8,9 años ( $\mathrm{DE}=2,6) ; 82,5 \%$ residían en estrato I y el 17,5\% restante, en estrato II; 84,5\% eran amas de casa; 42\% eran nulíparas y en el 38,5\% era el primer embarazo. El número de gestaciones se encontró entre 1 y 8 , con un promedio de 2,7 $(\mathrm{DE}=9,8)$; la edad gestacional estuvo entre 5 y 40 semanas con un promedio de 26,8 semanas $(\mathrm{DE}=9,5)$ y $57,5 \%$ no planificaron el embarazo actual.

El instrumento para validar, la escala de Edinburgh para depresión posparto, consta de diez ítems de respuesta politómica (cuatro opciones de respuesta) que exploran síntomas cognoscitivos de un episodio depresivo mayor durante los últimos quince días. Cada pregunta se califica de cero a tres puntos. Requiere únicamente cinco a diez minutos para diligenciarse. La escala completa se presenta en la tabla $1 .^{13}$ 
Tabla 1. Escala de Edinburgh para depresión posparto.

He podido reír y apreciar lo divertido de las cosas He disfrutado las cosas que hago Me he echado la culpa innecesariamente cuando las cosas no han salido bien Me he sentido preocupada y ansiosa sin una buena razón

Me he sentido asustada o en pánico sin una buena razón

He sentido que las cosas se me están viniendo encima He estado tan infeliz que tengo problemas para dormir

Me he sentido triste o miserable

He estado tan infeliz que me he puesto a llorar Se me ha ocurrido la posibilidad de hacerme daño a mí misma

En la escala de Edinburgh para depresión posparto se determinó la consistencia interna mediante la prueba alfa de Cronbach, ${ }^{29}$ la correlación corregida ítem-puntuación total mediante la correlación de Pearson $(r)^{30}$ y el alfa de Cronbach, si el ítem se omitiera. Para explorar la estructura factorial se realizó un análisis de factores exploratorio mediante el método de componentes principales. Esto permite conocer los factores o dominios latentes en una escala lo suficientemente homogénea y aproximarse a la validez del constructo. ${ }^{20,21}$ Inicialmente, se aplicó la prueba de adecuación de la muestra de KayserMeyer-Olkin $(\mathrm{KMO})^{31}$ y la prueba de esfericidad de Bartlett. ${ }^{32}$ Se consideraron factores importantes los que mostraron valores propios mayores de 1,0 y por lo menos tres ítems con coeficientes mayores de 0,50. ${ }^{20,21}$ La solución final se rotó en forma oblicua (promax) porque se asumió con anticipación que los factores estarían altamente correlacionados. Los datos se procesaron por duplicado para identificar inconsistencias. Todos los cálculos se realizaron en el paquete estadístico SPSS 13.0 para Windows. ${ }^{33}$

\section{RESULTADOS}

Se encontró que la consistencia interna de la escala de Edinburgh para depresión posparto fue 0,78. Las correlaciones corregidas ítem-puntuación total se muestran en la tabla 2. La prueba de esfericidad de Bartlett mostró un $\mathrm{X}^{2}=495,1 \mathrm{gl}$ $=45 \mathrm{p}<0,001$ y una prueba de KMO de 0,81. Estos coeficientes indican que debe rechazarse la hipótesis de la matriz identidad. La escala de Edinburgh para depresión posparto mostró una estructura tridimensional que explicaba el 59,9\% de la varianza. A los factores se les denominó basados en la clasificación teórica de los síntomas depresivos y por los valores de los coeficientes en la matriz factorial. El primer factor (ánimo deprimido) mostró un valor propio de 3,54 que daba cuenta el 35,4\% de la varianza, el segundo factor (anhedonia) de 1,36 que explicaba el 13,6\% de la varianza y en tercer factor (desesperanza y culpa) de 1,10 responsable del $11,0 \%$ de la varianza. Ver detalles en la tabla 3.

\begin{tabular}{lcc}
$\begin{array}{l}\text { Tabla 2. Confiabilidad de la escala de Edinburgh } \\
\text { para depresión posparto en gestantes estrato } \\
\text { bajo en Cartagena, Colombia, } 2007 .\end{array}$ \\
\multicolumn{1}{c}{ Ítem } & i-t** & $\begin{array}{c}\text { Alfa de Cronbach } \\
\text { si el ítem se omite }\end{array}$ \\
Reír & 0,46 & 0,76 \\
Disfrutar & 0,44 & 0,77 \\
Culpa & 0,37 & 0,77 \\
Ansiedad & 0,28 & 0,79 \\
Pánico & 0,51 & 0,76 \\
Desesperanza & 0,23 & 0,79 \\
Infelicidad & 0,55 & 0,75 \\
Triste & 0,66 & 0,74 \\
Llorar & 0,62 & 0,74 \\
Ideación suicida & 0,40 & 0,77
\end{tabular}

*Correlación ítem-puntuación total corregida.

\section{DISCUSIÓN}

Se puede observar en la presente investigación que la escala de Edinburgh para depresión posparto muestra una alta consistencia interna y una estructura tridimensional de síntomas depresivos en gestantes de estrato bajo de Cartagena, Colombia, lo que sugiere que la escala es confiable y posiblemente válida, para ser utilizada en la identificación de posibles 
Tabla 3. Matriz de correlación de la escala

de Edinburgh para depresión posparto con

rotación oblicua en gestantes de estrato bajo en Cartagena, Colombia, 2007.

\begin{tabular}{|lccc|}
\hline \multicolumn{1}{|c}{ Ítem } & Factor I & $\begin{array}{c}\text { Factor } \\
\text { II }\end{array}$ & $\begin{array}{c}\text { Factor } \\
\text { III }\end{array}$ \\
\hline Reír & 0,407 & $\mathbf{0 , 7 5 8}$ & 0,101 \\
\hline Disfrutar & 0,486 & $\mathbf{0 , 7 4 2}$ & $-0,063$ \\
\hline Culpa & 0,299 & 0,218 & $\mathbf{0 , 6 3 7}$ \\
Ansiedad & $-0,076$ & $\mathbf{0 , 7 3 1}$ & 0,400 \\
\hline Pánico & 0,412 & 0,397 & $\mathbf{0 , 6 3 0}$ \\
\hline Desesperanza & 0,147 & $-0,013$ & $\mathbf{0 , 7 3 1}$ \\
\hline Infelicidad & $\mathbf{0 , 7 1 9}$ & 0,245 & 0,439 \\
\hline Triste & $\mathbf{0 , 7 4 9}$ & $\mathbf{0 , 5 8 3}$ & 0,267 \\
\hline Llorar & $\mathbf{0 , 7 9 0}$ & 0,374 & 0,357 \\
\hline Ideación suicida & $\mathbf{0 , 7 0 4}$ & 0,171 & 0,112 \\
\hline
\end{tabular}

* Coeficientes mayores de 0,500 aparecen en negrita.

episodios depresivos mayores en esta población.

En relación con la consistencia interna, similar a lo observado en el presente estudio, Ortega y cols. informaron una consistencia interna de 0,$82 ;^{22}$ Lartigue y cols., 0,85; ${ }^{23}$ Espíndola y cols., 0,$85 ;^{24} \mathrm{y}$ Adouard y cols., $0,76 .^{25}$

En lo referente al análisis factorial, en esta investigación se observó una estructura tridimensional, a diferencia de lo encontrado por Ortega y cols., Lartigue y cols. y Adouard y cols. quienes informaron que la escala de Edinburgh para depresión posparto estaba compuesta de una estructura de dos dimensiones. ${ }^{22,23,25}$ Para Ortega et al. el primer factor agrupaba "síntomas depresivos" y el segundo, "síntomas de ansiedad", los cuales explicaban algo más del 50\% de la varianza; ${ }^{22}$ para Lartigue y cols., el primer factor "síntomas depresivos y de ansiedad" y el segundo "displacer e ideación suicida" que daban cuenta de aproximadamente el 54\% de la varianza, ${ }^{23}$ y para Adouard y cols. el primer factor reunía ánimo deprimido y anhedonia y el segundo, ansiedad y distorsiones cognoscitivas. ${ }^{25}$

Las diferencias observadas en cuanto a la estructura factorial de la escala de Edinburgh para depresión posparto probablemente son debidas a aspectos sociodemográficos y elementos culturales. Estas características pueden modificar el comportamiento psicométrico de una escala ${ }^{17}$. La población que participó en el estudio que se presenta residía solo en estratos uno y dos con características culturales y lingüísticas propias del Caribe colombiano, que pueden influir en la forma como se interpreta cada ítem. Sin duda, como sostienen Jomeen y Martin, la solución factorial multidimensional para la escala de Edinburgh para depresión posparto es más satisfactoria que la solución unidimensional originariamente sugerida en el estudio inicial de validación ${ }^{34}$.

Los resultados obtenidos sugieren que la escala de Edinburgh para depresión posparto es un instrumento confiable y posiblemente válido para ser utilizado en la detección temprana de trastorno depresivo mayor en la población de gestantes de estrato bajo de Cartagena. A pesar de que algunos ítems mostraron una modesta correlación corregida con la puntuación total, la omisión individual de los mismos no modificaba sustancialmente la consistencia interna de la escala. Se espera que la consistencia interna de una escala sea alta cuando los valores se encuentren entre 0,70 y $0,90 .^{17,18}$ Por su parte, es deseable que los factores retenidos de una escala estén formados por al menos tres ítems con altos coeficientes (generalmente mayor de 0,50 ) y que expliquen por lo menos el $50 \%$ de la varianza. ${ }^{20,21}$

La identificación de posibles episodios depresivos mayores tal vez pueda prevenir las consecuencias negativas asociadas a los mismos, tanto para la madre como para el feto, si se hace un tratamiento adecuado. ${ }^{4,8,11}$

Sin duda, debido a la sencillez y al reducido tiempo necesario para su aplicación (cinco a diez minutos), la escala de Edinburgh para depresión posparto permite su utilización por cualquier profesional de la salud y se convierte en un instrumento importante para la identificación de posibles trastornos depresivos durante el embarazo. Esto mejora de manera importante la atención en la salud de las embarazadas. 
Se debe tener en cuenta que el presente estudio posee limitaciones, como el contar con una muestra no probabilística, participantes solo de estrato uno y dos (probablemente con menores grados de escolaridad y habilidades en lectoescritura) y la falta de un patrón de oro para explorar otras propiedades psicométricas. Se ha anotado con anterioridad que el comportamiento psicométrico de la escala puede variar dramáticamente por las características de la población. . $^{1718}$

Se concluye que la escala de Edinburgh para depresión posparto es un instrumento con una adecuada consistencia interna y estructura factorial para ser utilizado en la detección temprana de episodio depresivo mayor en la población de gestantes de estrato bajo de Cartagena, Colombia.

\section{REFERENCIAS}

1. Gilchrist G, Gunn J. Observational studies of depression in primary care: what do we know? BMC Fam Pract 2007;8:28.

2. Gómez-Restrepo C, Bohórquez A, Pinto-Masis D, GilLaverde JF, Rondón-Sepúlveda M, Díaz-Granados N. Prevalencia de depresión y factores asociados con ella en la población colombiana. Rev Panam Salud Pública 2004;16:378-86.

3. Blazer DG, Kessler RC, McConagle KA, Swartz MS. The prevalence and distribution of major depression in a national community sample: the National Comorbidity Survey. Am J Psychiatry 1994;151:979-86.

4. Evans J, Heron J, Francomb H, Oke S, Golding J. Cohort study of depressed mood during pregnancy and after childbirth. BMJ 2001;323:257-60.

5. Josefsson A, Berg G, Nordin C, Sydsjö G. Prevalence of depressive symptoms in late pregnancy and postpartum. Acta Obstet Gynecol Scand 2001;80:251-5.

6. Chaudron LH, Kitzman HJ, Peifer KL, Morrow S, Perez L, Newman MC. Prevalence of maternal depressive symptoms in low-income Hispanic women. J Clin Psychiatry 2005;66:418-23.

7. O'Boyle AL, Magann EF, Ricks RE Jr, Doyle M, Morrison JC. Depression screening in the pregnant soldier wellness program. South Med J 2005;98:416-8.

8. Rahman A, Bunn J, Lovel H, Creed F. Association between antenatal depression and low birthweight in a developing country. Acta Psychiatr Scand 2007;115:481-6.

9. Alvarado R, Rojas M, Monardes J, Perucca E, Neves E, Olea E, et al. Cuadros depresivos en el posparto en una cohorte de embarazadas. Rev Chil Neuro-Psiquiat 2000;38:84-93.

10. Bowen A, Muhajarine N. Antenatal depression. Can Nurse 2006;102:26-30.

11. Condon JT, Corkindale C. The correlates of antenatal attachment in pregnant women. Br J Med Psychol 1997;70:359-72.

12. Radloff LS. The CES-D scale: a self-report depression scale for research in the general population. Applied Psychological Measurement 1977;1:385-401.

13. Cox JL, Holden JM, Sagovsky R. Detection of postnatal depression: Development of the 10-item Edinburgh postnatal depression scale. Br J Psychiatry 1987;150:782-6.

14. Cox JL, Holden J. Perinatal mental health: a guide to the Edinburgh postnatal depression scale. London: Gaskell; 2003. p. 15-20.

15. Bowen A, Muhajarine N. Prevalence of antenatal depression in women enrolled in an outreach program in Canada. J Obstet Gynecol Neonatal Nurs 2006;35:491-8.

16. Gordon TE, Cardone IA, Kim JJ, Gordon SM, Silver RK. Universal perinatal depression screening in an Academic Medical Center. Obstet Gynecol 2006;107:342-7.

17. Sánchez R, Echeverry J. Validación de escalas de medición en salud. Rev Salud Pública 2004;6:302-18.

18. Oviedo HC, Campo-Arias A. Aproximación al uso del coeficiente alfa de Cronbach. Rev Colomb Psiquiatr 2005;34:572-80.

19. Morgan GA, Gliner JA, Harmon RJ. Measurement validity. J Am Acad Child Adolesc Psychiatry 2001;40:729-31.

20. Streiner DL. Figuring out factors: the use and misuse of factor analysis. Can J Psychiatry 1994;39:135-40.

21. Gorsuch RL. Exploratory factor analysis: its role in item analysis. J Pers Asses 1997;68:532-60.

22. Ortega L, Lartigue T, Figueroa ME. Prevalencia de depresión, a través de la escala de depresión perinatal de Edinburgh (EPDS), en una muestra de mujeres mexicanas embarazadas. Perinatol Reprod Hum 2001;15:11-20. 
23. Lartigue T, Casanova G, Ortiz J, Aranda C, Flórez CE. Indicadores de malestar emocional y depresión en mujeres embarazadas con ITS-VIH/SIDA. Perinatol Reprod Hum 2004;18:73-90.

24. Espíndola JG, Morales F, Mota C, Díaz E, Meza P, Rodríguez L. Calibración del punto de corte para la escala de depresión perinatal de Edinburgh en pacientes obstétricas del Instituto Nacional de Perinatología. Perinatol Reprod Hum 2004;3:179-86.

25. Adouard F, Glangeaud-Freudenthal NMC, Golse B. Validation of the Edinburgh postnatal depression scale (EPDS) in a sample of women with high-risk pregnancies in France. Arch Womens Ment Health 2005;8:89-95.

26. Blacker D, Endicott J. Psychometric properties: concepts of reliability and validity. En: Rush AJ, Pincus HA, First MB, Zarin DA, Blacker D, Endicott J, et al. (eds). Handbook of psychiatric measures. Washington, DC: American Psychiatric Association; 2000. p. 7-14.

27. Declaración de Helsinki de la Asociación Médica
Mundial. Visitado en 2006 Feb 21. Disponible en: Disponible en: www.wma.net/s/policy/b3.htm

28. Republica de Colombia. Ministerio de Salud. Resolución 008430, por la cual se establecen las normas científicas, técnicas y administrativas para la investigación en salud; 1993.

29. Cronbach LJ. Coefficient alpha and the internal structure of test. Psychometrika 1951;16:297-334.

30. Pearson ES. Studies in the story of probability and statistics. XIV. Some incidents in the early history of biometry and statistics, 1880-94. Biometrika 1965;52:3-18.

31. Kaiser HF. An index of factorial simplicity. Psychometrika 1974;39:31-6.

32. Bartlett MS. Test of significance in factor analysis. $\mathrm{Br}$ J Psychol 1950;3:77-85.

33. SPSS for Windows 13.0. Chicago: SPSS Inc., 2005.

34. Jomeen J, Martin CR. Replicability and stability of the multidimensional model of the Edinburgh postnatal depression scale in late pregnancy. J Psychiatr Ment Health Nurs 2007;14:319-24.

Conflicto de intereses: este trabajo fue financiado con recursos de la Facultad de Enfermería de la Universidad de Cartagena, Colombia. 